\title{
Association of Polymorphisms in Toll-Like Receptors 4 and 9 with Autoimmune Thyroid Disease in Korean Pediatric Patients
}

\author{
Won Kyoung Cho, ${ }^{1}$ Jung-Pil Jang, ${ }^{2}$ Eun-Jeong Choi, ${ }^{3}$ Moonbae Ahn, ${ }^{4}$ Shin Hee Kim, ${ }^{5}$ \\ Kyoung Soon Cho, ${ }^{6}$ So Hyun Park, ${ }^{1}$ In Cheol Baek, ${ }^{2}$ Min Ho Jung, ${ }^{7}$ Tai-Gyu Kim, ${ }^{2,3}$ and \\ Byung-Kyu Suh ${ }^{4}$ \\ ${ }^{1}$ Department of Pediatrics, College of Medicine, St. Vincent's Hospital, The Catholic University of Korea, Seoul, Republic of Korea \\ ${ }^{2}$ Department of Microbiology, College of Medicine, The Catholic University of Korea, Seoul, Republic of Korea \\ ${ }^{3}$ Catholic Hematopoietic Stem Cell Bank, College of Medicine, The Catholic University of Korea, Seoul, Republic of Korea \\ ${ }^{4}$ Department of Pediatrics, College of Medicine, Seoul St. Mary's Hospital, The Catholic University of Korea, Seoul, Republic of Korea \\ ${ }^{5}$ Department of Pediatrics, College of Medicine, Incheon St. Mary's Hospital, The Catholic University of Korea, Seoul, \\ Republic of Korea \\ ${ }^{6}$ Department of Pediatrics, College of Medicine, Bucheon St. Mary's Hospital, The Catholic University of Korea, Seoul, \\ Republic of Korea \\ ${ }^{7}$ Department of Pediatrics, College of Medicine, Yeouido St. Mary's Hospital, The Catholic University of Korea, Seoul, \\ Republic of Korea
}

Correspondence should be addressed to Tai-Gyu Kim; kimtg@catholic.ac.kr and Byung-Kyu Suh; suhbk@catholic.ac.kr

Received 3 February 2017; Revised 13 May 2017; Accepted 25 July 2017; Published 20 August 2017

Academic Editor: Maria L. Dufau

Copyright (C) 2017 Won Kyoung Cho et al. This is an open access article distributed under the Creative Commons Attribution License, which permits unrestricted use, distribution, and reproduction in any medium, provided the original work is properly cited.

\begin{abstract}
Background. Toll-like receptors (TLRs) have been suggested to be associated with the development of AITD. Methods. Fifteen single-nucleotide polymorphisms in 7 TLR genes were analyzed in 104 Korean children (girls $=86$, boys $=18$ ) with AITD (Hashimoto disease $(\mathrm{HD})=44$, Graves' disease $(\mathrm{GD})=60$, thyroid-associated ophthalmopathy $(\mathrm{TAO})=29$, and non-TAO $=31$ ) with 183 controls. Results. GD showed higher frequencies of the TLR4 rs1927911 C allele than control. TAO showed a lower frequency of the TLR4 rs1927911 CT genotype and non-TAO showed a higher frequency of the TLR4 rs1927911 CC genotype than control. The frequency of the TLR9 rs187084 CC genotype in TAO was higher than that in non-TAO. GD females showed a higher frequency of the TLR4 rs10759932 T allele, rs1927911 CC genotype, and the $r s 1927911 \mathrm{C}$ allele than controls. GD males showed a higher frequency of the TLR4 rs10759932 CC genotype and $r s 1927911$ TT genotype and lower frequency of the rs1927911 CT genotype than control. The frequency of the TLR4 rs10759932 CC genotype, C allele and $r s 1927911$ TT genotype, and $\mathrm{T}$ allele in a GD female were lower than in a GD male. Conclusions. Our results suggest that TLR4 and 9 polymorphisms might contribute to the pathogenesis of GD and TAO.
\end{abstract}

\section{Introduction}

It has been suggested that autoimmune thyroid disease (AITD) may occur when genetically susceptible individuals are exposed to environmental triggers such as infection, iodine, and stress [1]. AITD are female predominant and the biology of sexual dimorphism in AITD is not clearly understood. Recently, much attention and research funding has been focused on gender-based differences in AITD. In Taiwan, nationwide cohort studies have reported that AITD, including Hashimoto disease (HD) and Graves' disease (GD), might be risk factors of developing thyroid, breast, and colon cancers later in life $[2,3]$. 
Toll-like receptors (TLRs) recognize a variety of pathogen-associated molecular patterns such as bacteria, viruses, fungi, and certain host-derived molecules [4]. TLRs enable the innate immune system and induce a cascade of effector responses. TLRs are type I transmembrane glycoproteins with an extracellular domain of numerous leucine-rich repeats and an intracellular region containing a Toll $I L-1$ receptor homology domain [5].

Previous disease association studies revealed the effect of TLRs on the development of chronic inflammatory disease, injury, and cancer [6]. TLRs including TLR3 and 4 have been described on thyrocytes and reported to be associated with AITD or inflammatory disease [7-9]. In murine macrophages, gender difference in the expression of TLR4 for bacterial LPS has been reported [10]. We have reported an association between TLR10 polymorphisms and AITD [11]. In addition, TLR9 polymorphisms have been reported to be associated with TAO in Taiwanese males [12].

Genetic susceptibility might be a greater concern in early onset of AITD than in late onset of the disease. In our previous study, we observed increased allele frequencies for $H L A-B^{*} 46, H L A-D R B 1^{*} 08$, and $H L A-C w^{*} 01$ in children with AITD than in the control group [13]. The statistical significance in our results were significantly stronger than any other study conducted on Korean adults [14]. The aforementioned strong statistical significance might suggest that early-onset AITD is more influenced by genetic factors than in late-onset cases. In this study, we investigated the potential associations of seven TLR genes (TLR1, 2, 3, 4, 5, 6, and 9) including 15 single-nucleotide polymorphisms (SNP) with AITD in Korean children. We also comprehensively analyzed the association of TLR genes with disease subgroups based on sex and thyroid-associated ophthalmopathy (TAO) of AITD.

\section{Subjects and Methods}

2.1. Participants. This study analyzed 104 patients diagnosed with AITD: 44 with HD and 60 with GD $(\mathrm{TAO}=29$, non$\mathrm{TAO}=31$ ), who were treated at pediatric endocrine clinics at Seoul St. Mary's Hospital between March 2009 and August 2014. Of these patients, 84 were in a previous study conducted by our research group [11]. The age of patients at study enrollment was $13.2 \pm 3.5$ years and the age at AITD diagnosis was $11.3 \pm 3.2$ years (Table 1 ).

The control group consisted of 183 healthy Korean adults without a history of AITD, who were staff members and students at the College of Medicine at the Catholic University of Korea. All participants provided informed consent for a genetic study. This study was approved by the Institutional Review Board (IRB) of The Catholic University of Korea (IRB number: KC09FISI0042).

HD was diagnosed when at least three of the following criteria established by Fisher et al. [15] were met: goiter, diffuse goiter and decreased radionuclide uptake during thyroid scan, circulating thyroglobulin or microsomal autoantibodies, and hormonal evidence of hypothyroidism. GD
TABle 1: Characteristics of 104 autoimmune thyroid disease patients.

\begin{tabular}{lc}
\hline Characteristics & \\
\hline Sex (F/M) & $86 / 18$ \\
Age (years) at diagnosis & $11.3 \pm 3.2$ \\
Age (years) at enrollment & $13.2 \pm 3.5$ \\
HD/GD & $44 / 60$ \\
HD condition at diagnosis & \\
Euthyroid state & $9(20.5 \%)$ \\
Subclinical hypothyroid state & $6(13.6 \%)$ \\
Overt hypothyroid state & $23(52.3 \%)$ \\
Hyperthyroid state & $6(13.6 \%)$ \\
HD patients on T4 replacement & $25(56.8 \%)$ \\
Class of TAO & \\
$0 \sim 1$ no sign only sign & 75 \\
2 soft tissue involvement & 7 \\
3 proptosis & 19 \\
4 extraocular muscle involvement & 3 \\
5 corneal involvement & 0 \\
6 sight loss & 0 \\
\hline
\end{tabular}

AITD: autoimmune thyroid diseases; HD: Hashimoto's disease; GD: Graves' disease; TAO: thyroid-associated ophthalmopathy.

was diagnosed based on clinical symptoms and biochemical confirmation of hyperthyroidism, including diagnosis of goiter, elevated radioactive iodine uptake, antibodies against the TSH receptor, and elevated thyroid hormone levels. Patients with other forms of autoimmune, hematologic, or endocrine diseases were excluded. TAO was diagnosed based on the presence of typical clinical features and classified according to the system recommended by the American Thyroid Association Committee [16, 17]. Patients with no symptoms or only a lid lag sign were included in the without-TAO group. Patients with soft tissue changes, proptosis, extraocular muscle dysfunction, or the latter two symptoms were considered to have an eye disease [18].

2.2. DNA Extraction. Genomic DNA was extracted from peripheral blood cells using AccuPrep Genomic DNA Extraction kits (Bioneer Corporation, Daejeon, Korea), according to the manufacturer's guidelines. The concentration of DNA solutions was adjusted to $100 \mathrm{ng} / \mu \mathrm{l}$ and used as polymerase chain reaction (PCR) templates for genotyping.

2.2.1. Analysis of TLR Polymorphisms. Genotyping was performed with using a direct sequence method. Fifteen SNPs of the 7 Toll-like receptor gene (TLR1, 2, 3, 4, 5, 6, and 9) were amplified by PCR using specific primers (Table 2). TLR4 has been described as a highly polymorphic gene [19]. The criteria the authors used to select the polymorphisms to be evaluated are as follows: First, genomic information of TLR4 was investigated (https://www.ncbi.nlm.nih.gov/gene/7099). Based on the aforementioned investigation, we reviewed articles on disease associations with TLR4 SNP. Among candidate's polymorphisms to be evaluated, we excluded 
TABLe 2: Primer sequences for each single-nucleotide polymorphism.

\begin{tabular}{|c|c|c|c|}
\hline Gene & SNP & Direction & Primer sequence $\left(5^{\prime} \rightarrow 3^{\prime}\right)$ \\
\hline \multirow{2}{*}{ TLR1 } & $r s 4833095$ & $\mathrm{~F}$ & GCCAAACCAGCTGGAGGATCC \\
\hline & $(+742)$ & $\mathrm{R}$ & TGGGGAACACAATGTGCAGACTC \\
\hline \multirow{6}{*}{ TLR2 } & rs4696480 & $\mathrm{F}$ & CAAGATTGAAGGGCTGCATCTGG \\
\hline & $(-16934)$ & $\mathrm{R}$ & CCACCTCTCAGCTCGCAGTGAG \\
\hline & rs1898830 & $\mathrm{F}$ & GAAGAGTGACGAAAAATGAATGAGCA \\
\hline & (intron1) & $\mathrm{R}$ & GATGAACCTCTGGCAAGACAATAAAAG \\
\hline & rs7656411 & $\mathrm{F}$ & GCCTGCCCTTTTTCссСTTC \\
\hline & $\left(3^{\prime} U T R\right)$ & $\mathrm{R}$ & TTAAGCTGGGAACCATGTGAAAGG \\
\hline \multirow{4}{*}{ TLR3 } & rs3775291 & $\mathrm{F}$ & TGGCCCAACCAAGAGAAAGCA \\
\hline & $(c .1243)$ & $\mathrm{R}$ & TGGGGAGTGAGGCAAGGGAA \\
\hline & rs3775296 & $\mathrm{F}$ & CCAATGCATTTGAAAGCCATCTG \\
\hline & $(-7)$ & $\mathrm{R}$ & CCTTTTGCCCTTTGGGATGC \\
\hline \multirow{6}{*}{ TLR4 } & rs11536889 & $\mathrm{F}$ & TGGGCAATGCTCCTTGACCAC \\
\hline & $\left(3^{\prime} U T R\right)$ & $\mathrm{R}$ & GGACAATCAGGATGTCATCAGGGA \\
\hline & rs10759932 & $\mathrm{F}$ & CACTTGCTACTTTCCAGACACTGTCCT \\
\hline & $(-1607)$ & $\mathrm{R}$ & TGAGAACTCCTGTACACCATTTGTGG \\
\hline & rs1927911 & $\mathrm{F}$ & TGGCCCAGATTTTGACAACTGC \\
\hline & (intron1) & $\mathrm{R}$ & CATGGATTCCCATGGTGGAACC \\
\hline \multirow[t]{2}{*}{ TLR5 } & rs5744168 & $\mathrm{F}$ & TCTGGGGGAACTTTACAGTTCGAA \\
\hline & $(+1174)$ & $\mathrm{R}$ & TGCCAAAGATCAACCTTACAGCG \\
\hline \multirow{4}{*}{ TLR6 } & rs5743810 & $\mathrm{F}$ & CССТTCACCTTGTTTTTCACCCA \\
\hline & $(+745)$ & $\mathrm{R}$ & CTTGGAAATGCCTGGTCAGAGTCT \\
\hline & rs2381289 & $\mathrm{F}$ & CTGCAAGGAAGGCCAAGCAGA \\
\hline & $\left(3^{\prime} U T R\right)$ & $\mathrm{R}$ & TGAAGCCCTCGCTTTCTGGACT \\
\hline \multirow{6}{*}{ TLR9 } & $r s 352140$ & $\mathrm{~F}$ & CGCTGACCGGTCTGCAGGT \\
\hline & $(+2848)$ & $\mathrm{R}$ & ACTGGAGGCCCTGGACCTCA \\
\hline & rs 187084 & $\mathrm{~F}$ & ACTGGATCCTGGGGATGCAGA \\
\hline & $(-1486)$ & $\mathrm{R}$ & AGCTGACATTCCAGCAGGGGA \\
\hline & $r s 352162$ & $\mathrm{~F}$ & TCTCCTGAATCTCCAGCCCCA \\
\hline & $\left(3^{\prime} U T R\right)$ & $\mathrm{R}$ & TGGCAATCCCAAGACAGGCA \\
\hline
\end{tabular}

SNP: single-nucleotide polymorphism; TLR: Toll-like receptor.

polymorphisms having MAF 1.0 in population diversity of Japanese in Tokyo (https://www.ncbi.nlm.nih.gov/variation/ tools/1000genomes/). In this process, some polymorphisms including TLR4 rs4986791 $(\mathrm{C}=1.000)$ and $r s 4986790$ $(\mathrm{A}=1.000)$ were ruled out. Finally, the disease associations of TLR4 rs1927911, rs10759932, and rs11536889 were evaluated. Other TLR genes were also determined in this way.

Amplification was performed in a GeneAmp PCR System 9700 thermocycler (Applied Biosystems, Foster City, CA, USA) using the following conditions: one cycle at $95^{\circ} \mathrm{C}$ for $5 \mathrm{~min}$ and 35 cycles of denaturation at $95^{\circ} \mathrm{C}$ for $30 \mathrm{sec}$, annealing at $55-62^{\circ} \mathrm{C}$ (depending on primer set) for $30 \mathrm{sec}$, extension at $72^{\circ} \mathrm{C}$ for $1 \mathrm{~min}$, and final extension at $72^{\circ} \mathrm{C}$ for $10 \mathrm{~min}$. The length of amplified products was confirmed by electrophoresis on $1.5 \%$ agarose gels.

PCR products from the second round were cleaned using exonuclease I and shrimp alkaline phosphatase (United States Biochemical) and used as sequencing templates.
Sequencing was performed using a Big Dye Terminator version 3.1 (Amersham Pharmacia) and reactions were analyzed with ABI PRISM 3730XL analyzer (PE Applied Biosystems, Foster City, CA, USA). Sequencing data was analyzed with FinchTV software version 1.4 (Geospiza Inc., Seattle, Washington, USA).

2.2.2. Statistical Analysis. Allele frequencies were determined using Microsoft Office Excel. For controls, Hardy-Weinberg equilibrium was analyzed for each single-nucleotide polymorphism (SNP) with SNPStats (http://bioinfo.iconcologia .net/snpstats/start.htm). Fisher's exact test was applied when expected frequency was lower than 5 . The $P$ value was multiplied by the number of alleles observed for corrected $P$ value $(P c)$ to account for multiple comparisons performed. A Pc value $<0.05$ was considered statistically significant. Haldane's formula correction was used when critical entries were equal to zero. 
TABLE 3: Allele frequencies of Toll-like receptor polymorphism in controls and patients with thyroid disease.

\begin{tabular}{|c|c|c|c|c|c|}
\hline & SNP alleles & $\begin{array}{c}\text { Normal } \\
2 n=366(\%)\end{array}$ & $\begin{array}{c}\text { AITD } \\
2 n=208(\%)\end{array}$ & $\begin{array}{c}\text { GD } \\
2 n=120(\%)\end{array}$ & $\begin{array}{c}\text { HD } \\
2 n=88(\%)\end{array}$ \\
\hline TLR1 (+742) & $\mathrm{C}$ & $235(64.2)$ & $126(60.6)$ & $74(61.7)$ & $52(59.1)$ \\
\hline rs4833095 & $\mathrm{T}$ & $131(35.8)$ & $82(39.4)$ & $46(38.3)$ & $36(40.9)$ \\
\hline TLR2 (-16934) & A & $187(51.1)$ & $115(55.3)$ & $65(54.2)$ & $50(56.8)$ \\
\hline$r s 4696480$ & $\mathrm{~T}$ & $179(48.9)$ & $93(44.7)$ & $55(45.8)$ & $38(43.2)$ \\
\hline TLR2 (intron1) & A & $185(50.5)$ & $112(53.8)$ & $66(55.0)$ & $46(52.3)$ \\
\hline rs1898830 & G & $181(49.5)$ & $96(46.2)$ & $54(45.0)$ & $42(47.7)$ \\
\hline TLR2 (3'UTR) & G & $180(49.2)$ & $116(55.8)$ & $70(58.3)$ & $46(52.3)$ \\
\hline$r s 7656411$ & $\mathrm{~T}$ & $186(50.8)$ & $92(44.2)$ & $50(41.7)$ & $42(47.7)$ \\
\hline TLR3 (c.1243) & A & $111(30.3)$ & $59(28.4)$ & $31(25.8)$ & $28(31.8)$ \\
\hline$r s 3775291$ & G & $255(69.7)$ & $149(71.6)$ & $89(74.2)$ & $60(68.2)$ \\
\hline $\operatorname{TLR} 3(-7)$ & A & $80(21.9)$ & $49(23.6)$ & $24(20.0)$ & $25(28.4)$ \\
\hline rs3775296 & $\mathrm{C}$ & $286(78.1)$ & $159(76.4)$ & $96(80.0)$ & $63(71.6)$ \\
\hline TLR4 (3'UTR) & $\mathrm{C}$ & $83(22.7)$ & $49(23.6)$ & $31(25.8)$ & $18(20.5)$ \\
\hline rs11536889 & G & $283(77.3)$ & $159(76.4)$ & $89(74.2)$ & $70(79.5)$ \\
\hline TLR4 (-1607) & $\mathrm{C}$ & $103(28.1)$ & $47(22.6)$ & $25(20.8)$ & $22(25.0)$ \\
\hline rs10759932 & $\mathrm{T}$ & $263(71.9)$ & $161(77.4)$ & $95(79.2)$ & $66(75.0)$ \\
\hline TLR4 (intron1) & $\mathrm{C}$ & $219(59.8)$ & $135(64.9)$ & $84(70.0)^{\mathrm{a}}$ & $51(58.0)$ \\
\hline$r s 1927911$ & $\mathrm{~T}$ & $147(40.2)$ & $73(35.1)$ & $36(30.0)$ & $37(42.0)$ \\
\hline CC-TLR5 (+1174) & $\mathrm{C}$ & $362(98.9)$ & $205(98.6)$ & $118(98.3)$ & 87 (98.9) \\
\hline rs5744168 & $\mathrm{T}$ & $4(1.1)$ & $3(1.4)$ & $2(1.7)$ & $1(1.1)$ \\
\hline CC-TLR6 $(+745)$ & $\mathrm{C}$ & $366(100.0)$ & $208(100.0)$ & $120(100.0)$ & $88(100.0)$ \\
\hline rs5743810 & G & $0(0.0)$ & $0(0.0)$ & $0(0.0)$ & $0(0.0)$ \\
\hline TLR6 (3'UTR) & $\mathrm{C}$ & $180(49.2)$ & $117(56.3)$ & $66(55.0)$ & $51(58.0)$ \\
\hline$r s 2381289$ & $\mathrm{~T}$ & $186(50.8)$ & $91(43.7)$ & $54(45.0)$ & $37(42.0)$ \\
\hline TLR9 $(+2848)$ & A & $150(41.0)$ & $89(42.8)$ & $50(41.7)$ & $39(44.3)$ \\
\hline$r s 352140$ & G & $216(59.0)$ & $119(57.2)$ & $70(58.3)$ & $49(55.7)$ \\
\hline TLR9 $(-1486)$ & $\mathrm{C}$ & $152(41.5)$ & $91(43.8)$ & $51(42.5)$ & $40(45.5)$ \\
\hline rs187084 & $\mathrm{T}$ & $214(58.5)$ & $117(56.2)$ & $69(57.5)$ & $48(54.5)$ \\
\hline TLR9 (3'UTR) & $\mathrm{C}$ & $153(41.8)$ & $90(43.3)$ & $50(41.7)$ & $40(45.5)$ \\
\hline$r s 352162$ & $\mathrm{~T}$ & $213(58.2)$ & $118(56.7)$ & $70(58.3)$ & $48(54.5)$ \\
\hline
\end{tabular}

SNP: single-nucleotide polymorphism; AITD: autoimmune thyroid diseases; HD: Hashimoto's disease; GD: Graves' disease; TLR: Toll-like receptor. Controls versus GD: ${ }^{\mathrm{a}} \mathrm{OR}=1.56(1.0-2.4), P=0.046$.

\section{Results}

Allele frequencies of 15 SNPs in AITD and controls are in Table 3. For overall AITD, the allele frequencies of total TLR genes were not significantly different with controls. When AITD were categorized by the disease subgroup, GD showed higher frequencies of TLR4 rs1927911 C allele $(\mathrm{OR}=1.56 ; 95 \% \mathrm{CI}, 1.0-2.4, P=0.046)$ than those by the control group (Table 3 ).

When GD was categorized by TAO, the TAO group showed lower frequency of the TLR4 rs1927911 CT genotype $(\mathrm{OR}=0.4 ; 95 \% \mathrm{CI}, 0.18-1.00, P=0.047)$ and the non-TAO group showed a higher frequency of the rs1927911 CC genotype $(\mathrm{OR}=2.31 ; 95 \% \mathrm{CI}, 1.07-4.99, P=0.029)$ than the control group. Between the non-TAO and TAO groups, the frequency of the TLR9 $r s 187084$ CC genotype $(\mathrm{OR}=5.52$; 95\% CI, 1.06-28.7, $P=0.028$ ) in the TAO group was higher than that in the non-TAO group (Table 4).
When GD was categorized by sex, GD females showed a higher frequency of the TLR4 $r$ s10759932 T allele $(\mathrm{OR}=2.06$; 95\% CI, 1.13-3.74, $P=0.015, P \mathrm{C}=0.03), r s 1927911$ CC genotype $(\mathrm{OR}=2.36 ; 95 \% \mathrm{CI}, 1.23-4.52, P=0.008, P \mathrm{c}=0.026)$, and $r s 1927911 \mathrm{C}$ allele $(\mathrm{OR}=1.96 ; 95 \% \mathrm{CI}, 1.18-3.26$, $P=0.009, P c=0.018)$ than controls. GD males showed a higher frequency of the TLR4 rs10759932 CC genotype $(\mathrm{OR}=4.34 ; 95 \% \mathrm{CI}, 1.21-15.60, P=0.031)$ and $r s 1927911$ TT genotype $(\mathrm{OR}=3.61 ; 95 \% \mathrm{CI}, 1.1-11.87, P=0.033)$ and lower frequency of the $r s 1927911 \mathrm{CT}$ genotype $(\mathrm{OR}=0.18$; 95\% CI, 0.04-0.82, $P=0.011, P C=0.032)$ than controls (Table 5).

Between females and males in GD, the frequency of the TLR4 rs10759932 CC genotype (OR $=24$; 95\% CI, 2.52228.3, $P=0.002, P c=0.006), C$ allele $(\mathrm{OR}=3.29 ; 95 \% \mathrm{CI}$, 1.26-8.63, $P=0.012, P c=0.025)$, and TLR4 rs1927911 TT genotype $(\mathrm{OR}=9.17 ; \quad 95 \% \quad \mathrm{CI}, \quad 1.82-46.20, \quad P=0.008$, $P \mathrm{C}=0.024), \quad \mathrm{T}$ allele $(\mathrm{OR}=2.5 ; \quad 95 \% \quad \mathrm{CI}, \quad 1.02-6.15$, 
TABLE 4: Genotype frequencies of TLR4 genes between controls and patients with or without thyroid-associated ophthalmopathy in Graves' disease.

\begin{tabular}{lcccc}
\hline & & $\begin{array}{c}\text { Normal } \\
n=183(\%)\end{array}$ & $\begin{array}{c}\text { GD TAO } \\
n=29(\%)\end{array}$ & $\begin{array}{c}\text { GD non-TAO } \\
n=31(\%)\end{array}$ \\
\hline TLR4 (intron1) & CC & $63(34.4)$ & $15(51.7)$ & $17(54.8)^{\mathrm{b}}$ \\
rs1927911 & CT & $93(50.8)$ & $9(31.0)^{\mathrm{a}}$ & $11(35.5)$ \\
& TT & $27(14.8)$ & $5(17.2)$ & $3(9.7)$ \\
& $\mathrm{C}$ & $219(59.8)$ & $39(67.2)$ & $45(72.6)$ \\
& $\mathrm{T}$ & $147(40.2)$ & $19(32.8)$ & $17(27.4)$ \\
\hline TLR9 (-1486) & $\mathrm{CC}$ & $35(19.1)$ & $8(27.6)$ & $2(6.5)^{\mathrm{c}}$ \\
rs187084 & $\mathrm{CT}$ & $82(44.8)$ & $12(41.4)$ & $19(61.3)$ \\
& $\mathrm{TT}$ & $66(36.1)$ & $9(31.0)$ & $10(32.3)$ \\
& $\mathrm{C}$ & $152(41.5)$ & $28(48.3)$ & $23(37.1)$ \\
& $\mathrm{T}$ & $214(58.5)$ & $30(51.7)$ & $39(62.9)$ \\
\hline
\end{tabular}

AITD: autoimmune thyroid diseases; GD: Graves' disease; TAO: thyroidassociated ophthalmopathy. Controls versus TAO: ${ }^{\mathrm{a}} \mathrm{OR}=0.4(0.18-1.00)$, $P=0.047$; controls versus non-TAO: ${ }^{\mathrm{b}} \mathrm{OR}=2.31 \quad(1.07-4.99), P=0.029$; TAO versus non-TAO: ${ }^{\mathrm{C}} \mathrm{OR}=5.52(1.06-28.7), P=0.028$.

$P=0.042)$ in GD female was lower than that in GD male (Figure 1).

\section{Discussions}

In the present study, we found significant differences in genotype frequencies of TLR4 gene polymorphisms in patients with GD. For GD, the TLR4 rs1927911 C allele showed a disease-susceptible gene. The TLR4 gene, located at chromosome 9q32-q33, recognizes lipopolysaccharides (LPS) of gram-negative bacteria and fusion proteins and envelope proteins of viruses as ligands. It is surface expressed and recognizes extracellular ligands and microorganisms [20]. Previous studies have reported that the disease associations of TLR4 with chronic inflammatory disease include atherosclerosis, asthma, and rheumatoid arthritis [21]. It has been discovered that there is an association of TLR4 rs1927911 SNP with childhood asthma [22] and disease activity of rheumatoid arthritis [23] and type 2 diabetes mellitus [24]. Nicola et al. reported that all components of the LPS receptor complex are expressed on thyrocyte, and they also detected that thyroid cells recognize and respond to LPS using Fisher rat thyroid cell line-5 cells [8]. Combined with aforementioned evidence, we can suggest that TLR4 SNP could affect the pathogenesis of GD.

When GD was analyzed by TAO and compared with the control group, the TLR4 rs1927911 CC and CT showed a significant protective genotype for TAO. TLR4 signals via both the MyD88-independent pathway and MyD88-dependent pathways lead to robust IL-12 production, secretion of type I IFNs, and a string of Th1-type cellular and humoral immune responses [25]. We reported that the $I L-12$ gene could be involved in the pathogenesis of TAO in Korean children [26]. The present study has a similar purpose in terms of investigating the immunogenetics of Korean AITD adolescents, but the specific target genes are clearly different with our previous research report. Dysregulation of the
TABLE 5: Genotype frequencies of TLR4 genes between controls and female or male in GD.

\begin{tabular}{lcccc}
\hline & & $\begin{array}{c}\text { Normal } \\
n=183(\%)\end{array}$ & $\begin{array}{c}\text { GD female } \\
n=47(\%)\end{array}$ & $\begin{array}{c}\text { GD male } \\
n=13(\%)\end{array}$ \\
\hline TLR4 (-1607) & CC & $17(9.3)$ & $0(0.0)$ & $4(30.8)^{\mathrm{d}}$ \\
rs10759932 & CT & $69(37.7)$ & $15(31.9)$ & $2(15.4)$ \\
& TT & $97(53.0)$ & $32(68.1)$ & $7(53.8)$ \\
& C & $103(28.1)$ & $15(16.0)$ & $10(38.5)$ \\
& T & $263(71.9)$ & $79(84.0)^{\mathrm{a}}$ & $16(61.5)$ \\
\hline TLR4 (intron1) & CC & $63(34.4)$ & $26(55.3)^{\mathrm{b}}$ & $6(46.2)$ \\
rs1927911 & CT & $93(50.8)$ & $18(38.3)$ & $2(15.4)^{\mathrm{e}}$ \\
& TT & $27(14.8)$ & $3(6.4)$ & $5(38.5)^{\mathrm{f}}$ \\
& $\mathrm{C}$ & $219(59.8)$ & $70(74.5)^{\mathrm{c}}$ & $14(53.8)$ \\
& $\mathrm{T}$ & $147(40.2)$ & $24(25.5)$ & $12(46.2)$ \\
\hline
\end{tabular}

AITD: autoimmune thyroid diseases; GD: Graves' disease. Controls versus GD female: ${ }^{\mathrm{a}} \mathrm{OR}=2.06(1.13-3.74), P=0.015, P \mathrm{c}=0.03$; ${ }^{\mathrm{b}} \mathrm{OR}=2.36(1.23-$ 4.52), $P=0.008, P \mathrm{c}=0.026 ;{ }^{\mathrm{c}} \mathrm{OR}=1.96(1.18-3.26), P=0.009, P \mathrm{c}=0.018$; controls versus GD male: ${ }^{\mathrm{d}} \mathrm{OR}=4.34(1.21-15.60), P=0.031 ;{ }^{\mathrm{e}} \mathrm{OR}=0.18$ (0.04-0.82), $P=0.011, P \mathrm{c}=0.032 ;{ }^{\mathrm{f}} \mathrm{OR}=3.61(1.1-11.87), P=0.033$.

TLR4 signaling owing to SNPs may alter the ligand binding and balance between pro- and anti-inflammatory cytokines, thereby modulating the risk of chronic inflammation [27]. Some reports suggest TLR4 rs4986790 SNP and differences in LPS responsiveness in humans [28] and attenuated receptor signaling and diminished the inflammatory response to gram-negative pathogens [29]. The associations between TLR4 rs10759931 SNP and TLR4 expression in colon cancer tissues [30], TLR2-196 to TLR2-174del SNP, and TLR2 mRNA expression have been reported [31]. Variants in TLR2 and TLR4 were associated with monocyte receptor levels of TLR2 and TLR4, respectively, in a biracial cohort of adults [32]. Based on previous evidence, we may propose that TLR4 SNPs are associated with TAO because of a process in the activation of immune cell signaling through cytokine production. However, studies on the possible consequences of TLR4 SNPs in the function of the receptor are lacking and further large scale, well-designed, comprehensive studies are necessary in the future.

Between TAO and non-TAO, the frequency of the TLR9 rs187084 CC genotype in non-TAO is lower than that in TAO. TLR9 gene, located chromosome $3 \mathrm{p} 21.3$, recognizes CpG-containing DNA and DNA sugar backbone as ligand and is expressed in immune cells in intracellular endosomal compartments. Disease associations of TLR9 with SLE, type $1 \mathrm{DM}$, multiple sclerosis, inflammatory bowel disease, and rheumatoid arthritis have been reported [21]. In 2010, Liao et al. reported that the frequency of the TLR9 rs 187084 CC genotype in non-TAO $(6.25 \%)$ was lower than that in TAO (7.4\%) in Taiwanese males [12]. Therefore, the results of our study are similar to those of the study conducted in Taiwanese patients. Based on previous evidence, we suspected that TLR9 rs 187084 SNPs are associated with TAO.

AITD prevalence is female predominant and the ratio of female and male is approximately $7: 3$ [33]. Females generate more robust humoral and cell-mediated immune responses after following antigenic challenge than males. These 


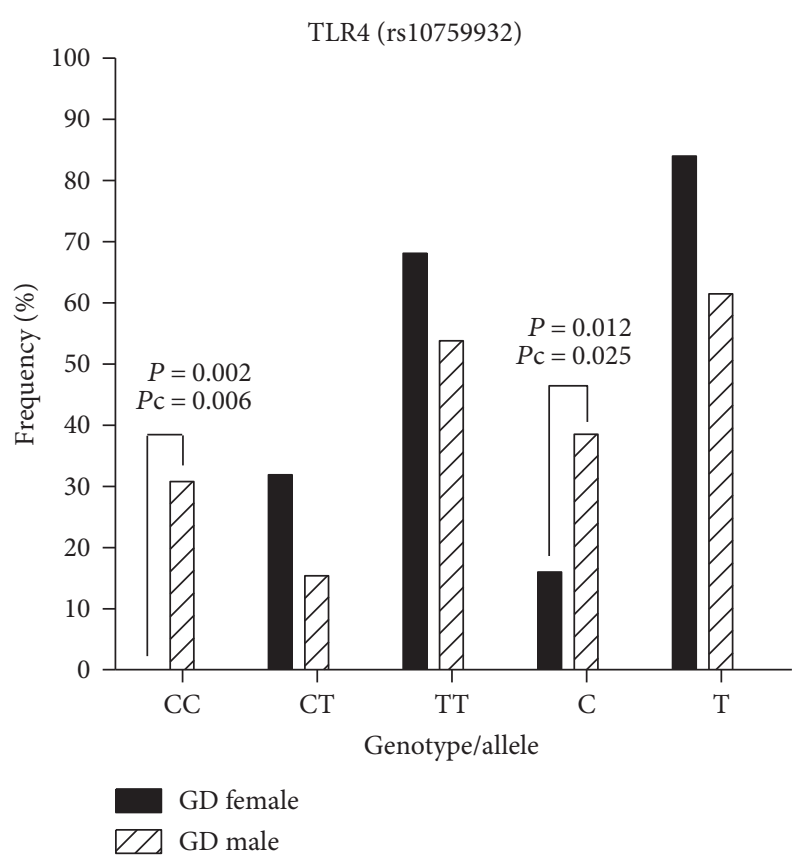

(a)

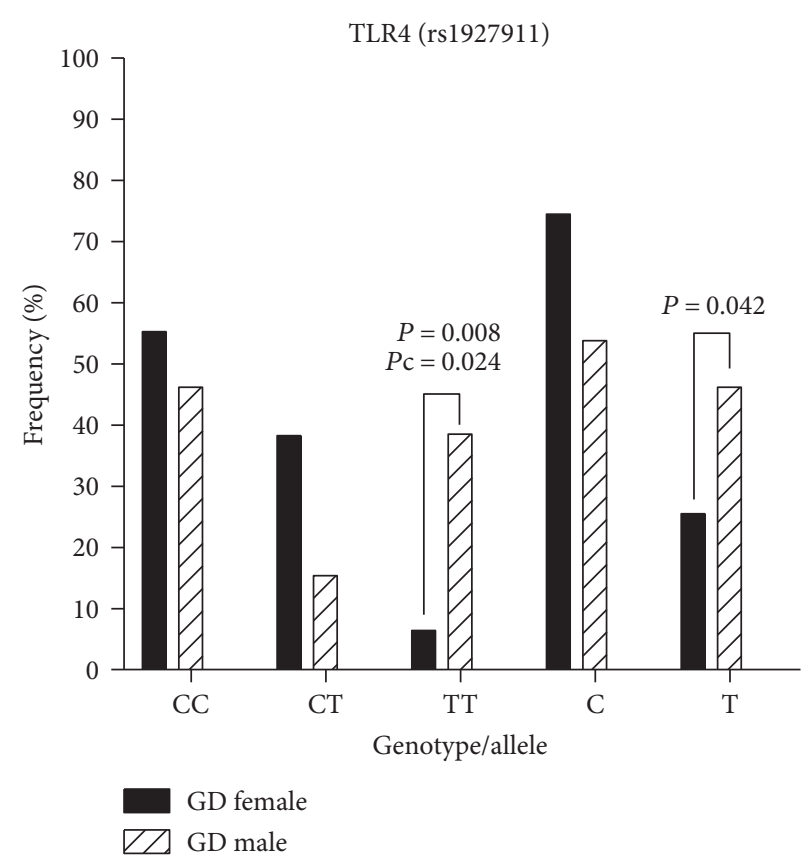

(b)

FIGURE 1: Analysis of TLR4 gene polymorphism between female and male in Graves' disease (GD); the frequency of the TLR4 rs 10759932 CC genotype $(\mathrm{OR}=24 ; 95 \% \mathrm{CI}, 2.52-228.3, P=0.002, P \mathrm{c}=0.006), \mathrm{C}$ allele $(\mathrm{OR}=3.29 ; 95 \% \mathrm{CI}, 1.26-8.63, P=0.012, P \mathrm{c}=0.025)$, and $T L R 4$ $r s 1927911$ TT genotype $(\mathrm{OR}=9.17 ; 95 \% \mathrm{CI}, 1.82-46.20, P=0.008, P \mathrm{c}=0.024)$; and $\mathrm{T}$ allele $(\mathrm{OR}=2.5 ; 95 \% \mathrm{CI}, 1.02-6.15, P=0.042)$ in GD female was lower than that in GD male.

elevated immune responses in females may underlie the higher incidence of an array of disorders thought to be autoimmune in origin. However, the biology of sexual dimorphism in autoimmune disease is not clearly understood. Previous reports suggest that $\mathrm{X}$ chromosome inactivation is an important contributor to the increased risk of females for developing AITD [34-36]. Sexual dimorphism in the expression of TLR4 for bacterial LPS in murine macrophages has been reported, and TLR4 might contribute to the greater susceptibility of males to bacteria sepsis [10]. In this study, we observed that GD females showed a higher frequency of the TLR4 rs10759932 $\mathrm{T}$ allele, rs1927911 CC genotype, and rs 192791 C allele than controls. GD males showed a higher frequency of the TLR4 rs10759932 CC and rs1927911 TT genotypes and a lower frequency of the $r s 1927911$ CT genotype than controls. Between GD male and GD female, the frequency of the TLR4 rs10759932 CC genotype, C allele, TLR4 rs1927911 TT genotype, and T allele was lower. TLR4 rs10759932 SNP has been reported to be associated with childhood asthma [37] and psoriasis vulgaris [38]. These results might suggest that TLR4 polymorphisms might influence the female predominance of GD and act as evidence explaining AITD pathogenesis.

TLR3 overexpression in thyrocytes from patients with HD has been reported, but not in normal thyrocytes or patients with GD. TLR3 overexpression induces an innate immune response in thyrocytes, which may be important in HD pathogenesis and in immune cell infiltrates [7]. Several TLR1, 2, and 6 polymorphisms have been described with functional and genetic association studies including asthma, rheumatoid arthritis, and inflammatory bowel disease
$[6,21]$. In this study, we tried to investigate the association between TLR1 rs4833095, TLR2 rs4696480, rs1898830, rs7656411, TLR3 rs3775291, rs3775296, TLR5 rs5744168, TLR6 rs5743810, rs 2381289, and AITD. However, there were no significant differences in genotype frequencies of TLR1, 2, 3, 5, and 6 between AITD and controls.

There are some limitations in this study. First, the control group in this research consisted mainly of both teaching and nonteaching staff and students from the College of Medicine at the Catholic University of Korea. The entire population of healthy control patients (adult population) is different from that of patients under analysis (pediatric population). Because there is no report that the distribution of HLA genotypes varies with age in a single race, the healthy controls of the adult population were used in this study despite the difference from that of patients under analysis (pediatric population). Furthermore, it is difficult to obtain enough pediatric control for ethical reason. Second, this study has a small number of cases and controls. Although there was limitation in getting a sufficient amount of samples, especially, pediatric AITD patient's samples, we were able to demonstrate the significant genetic associations of HLA, MICA, TLR10, and cytokine with AITD in pediatric patients which was conducted with a similar number of patient's samples as in this study $[11,13,26,39]$.

In conclusion, we suggest that TLR4 SNP may be involved in the pathogenesis of GD and TLR9 SNP could affect the pathogenesis of TAO. We also observed sexual dimorphism in the TLR4 gene in GD. Our data could be also used as baseline data for understanding the pathophysiology of AITD. 


\section{Conflicts of Interest}

The authors have declared no competing interests.

\section{Acknowledgments}

This study was supported by the Research Fund of Seoul St. Mary's Hospital, The Catholic University of Korea.

\section{References}

[1] Y. Tomer and T. F. Davies, "Searching for the autoimmune thyroid disease susceptibility genes: from gene mapping to gene function," Endocrine Reviews, vol. 24, no. 5, pp. 694717, 2003.

[2] Y. K. Chen, C. L. Lin, F. T. Cheng, F. C. Sung, and C. H. Kao, "Cancer risk in patients with Hashimoto's thyroiditis: a nationwide cohort study," British Journal of Cancer, vol. 109, no. 9, pp. 2496-2501, 2013.

[3] Y. K. Chen, C. L. Lin, Y. J. Chang et al., "Cancer risk in patients with Graves' disease: a nationwide cohort study," Thyroid, vol. 23, no. 7, pp. 879-884, 2013.

[4] B. Beutler, "Inferences, questions and possibilities in toll-like receptor signalling," Nature, vol. 430, no. 6996, pp. 257-263, 2004.

[5] R. J. Ulevitch, "Therapeutics targeting the innate immune system," Nature Reviews Immunology, vol. 4, no. 7, pp. 512-520, 2004.

[6] S. K. Drexler and B. M. Foxwell, "The role of toll-like receptors in chronic inflammation," International Journal of Biochemistry and Cell Biology, vol. 42, no. 4, pp. 506-518, 2010.

[7] N. Harii, C. J. Lewis, V. Vasko et al., "Thyrocytes express a functional toll-like receptor 3: overexpression can be induced by viral infection and reversed by phenylmethimazole and is associated with Hashimoto's autoimmune thyroiditis," Molecular Endocrinology, vol. 19, no. 5, pp. 1231-1250, 2005.

[8] J. P. Nicola, M. L. Vélez, A. M. Lucero, L. Fozzatti, C. G. Pellizas, and A. M. Masini-Repiso, "Functional toll-like receptor 4 conferring lipopolysaccharide responsiveness is expressed in thyroid cells," Endocrinology, vol. 150, no. 1, pp. 500-508, 2009.

[9] N. Inoue, M. Watanabe, Y. Katsumata, N. Ishido, Y. Hidaka, and Y. Iwatani, "Association between functional polymorphisms in the toll-like receptor 4 (TLR4) gene and HD severity," Tissue Antigens, vol. 85, no. 3, pp. 209-211, 2015.

[10] I. Marriott and Y. M. Huet-Hudson, "Sexual dimorphism in innate immune responses to infectious organisms," Immunologic Research, vol. 34, no. 3, pp. 177-192, 2006.

[11] W. K. Cho, J. P. Jang, E. J. Choi et al., “Association of Toll-like receptor 10 polymorphisms with autoimmune thyroid disease in Korean children," Thyroid, vol. 25, no. 2, pp. 250-255, 2015.

[12] W. L. Liao, R. H. Chen, H. J. Lin et al., "Toll-like receptor gene polymorphisms are associated with susceptibility to Graves' ophthalmopathy in Taiwan males," BMC Medical Genetics, vol. 11, p. 154, 2010.

[13] W. K. Cho, M. H. Jung, E. J. Choi, H. B. Choi, T. G. Kim, and B. K. Suh, "Association of HLA alleles with autoimmune thyroid disease in Korean children," Hormone Research in Poediatrics, vol. 76, no. 5, pp. 328-334, 2011.

[14] B. Y. Cho, B. D. Rhee, D. S. Lee et al., "HLA and Graves' disease in Koreans," Tissue Antigens, vol. 30, no. 3, pp. 119-121, 1987.
[15] D. A. Fisher, T. H. Oddie, D. E. Johnson, and J. C. Nelson, "The diagnosis of Hashimoto's thyroiditis," Journal of Clinical Endocrinology and Metabolism, vol. 40, no. 5, pp. 795-801, 1975.

[16] S. C. Werner, "Modification of the classification of the eye changes of Graves' disease: recommendations of the ad hoc committee of the American Thyroid Association," Journal of Clinical Endocrinology and Metabolism, vol. 44, no. 1, pp. 203-204, 1977.

[17] S. C. Werner, "Modification of the classification of the eye changes of Graves' disease," American Journal of Ophthalmology, vol. 83, no. 5, pp. 725-727, 1977.

[18] M. Frecker, V. Stenszky, C. Balazs, L. Kozma, E. Kraszits, and N. R. Farid, "Genetic factors in Graves' ophthalmopathy," Clinical Endocrinology, vol. 25, no. 5, pp. 479-485, 1986.

[19] M. Noreen, M. A. Shah, S. M. Mall et al., "TLR4 polymorphisms and disease susceptibility," Inflammation Research, vol. 61 , no. 3, pp. 177-188, 2012.

[20] S. Akira and K. Takeda, "Toll-like receptor signalling," Nature Reviews Immunology, vol. 4, no. 7, pp. 499-511, 2004.

[21] E. A. Misch and T. R. Hawn, "Toll-like receptor polymorphisms and susceptibility to human disease," Clinical Science (London, England: 1979), vol. 114, no. 5, pp. 347-360, 2008.

[22] E. Lee, J. W. Kwon, H. B. Kim et al., "Association between antibiotic exposure, bronchiolitis, and TLR4 (rs1927911) polymorphisms in childhood asthma," Allergy, Asthma \& Immunology Research, vol. 7, no. 2, pp. 167-174, 2015.

[23] M. L. Davis, T. D. LeVan, F. Yu et al., "Associations of toll-like receptor (TLR)-4 single nucleotide polymorphisms and rheumatoid arthritis disease progression: an observational cohort study," International Immunopharmacology, vol. 24, no. 2, pp. 346-352, 2015.

[24] Y. Xu, Z. Jiang, J. Huang, Q. Meng, P. Coh, and L. Tao, "The association between toll-like receptor 4 polymorphisms and diabetic retinopathy in Chinese patients with type 2 diabetes," British Journal of Ophthalmology, vol. 99, no. 9, pp. 13011305, 2015.

[25] D. N. Toussi and P. Massari, "Immune adjuvant effect of molecularly-defined toll-like receptor ligands," Vaccines (Basel), vol. 2, no. 2, pp. 323-353, 2014.

[26] J. P. Jang, W. K. Cho, I. C. Baek et al., "Comprehensive analysis of cytokine gene polymorphisms defines the association of IL12 gene with ophthalmopthy in Korean children with autoimmune thyroid disease," Molecular and Cellular Endocrinology, vol. 426, pp. 43-49, 2016.

[27] A. G. Kutikhin, "Impact of toll-like receptor 4 polymorphisms on risk of cancer," Human Immunology, vol. 72, no. 2, pp. 193-206, 2011.

[28] N. C. Arbour, E. Lorenz, B. C. Schutte et al., "TLR4 mutations are associated with endotoxin hyporesponsiveness in humans," Nature Genetics, vol. 25, no. 2, pp. 187-191, 2000.

[29] S. Kiechl, E. Lorenz, M. Reindl et al., "Toll-like receptor 4 polymorphisms and atherogenesis," New England Journal of Medicine, vol. 347, no. 3, pp. 185-192, 2002.

[30] A. Semlali, N. Reddy Parine, M. Arafah et al., "Expression and polymorphism of toll-like receptor 4 and effect on NF-kappaB mediated inflammation in colon cancer patients," PLoS One, vol. 11, no. 1, article e0146333, 2016.

[31] M. A. Proenca, J. G. de Oliveira, A. C. Cadamuro et al., “TLR2 and TLR4 polymorphisms influence mRNA and protein 
expression in colorectal cancer," World Journal of Gastroenterology, vol. 21, no. 25, pp. 7730-7741, 2015.

[32] S. J. Bielinski, J. L. Hall, J. S. Pankow et al., "Genetic variants in TLR2 and TLR4 are associated with markers of monocyte activation: the atherosclerosis risk in communities MRI study," Human Genetics, vol. 129, no. 6, pp. 655-662, 2011.

[33] C. C. Whitacre, "Sex differences in autoimmune disease," Nature Immunology, vol. 2, no. 9, pp. 777-780, 2001.

[34] X. Yin, R. Latif, Y. Tomer, and T. F. Davies, “Thyroid epigenetics: $\mathrm{X}$ chromosome inactivation in patients with autoimmune thyroid disease," Annals of the New York Academy of Sciences, vol. 1110, pp. 193-200, 2007.

[35] G. Chabchoub, E. Uz, A. Maalej et al., "Analysis of skewed Xchromosome inactivation in females with rheumatoid arthritis and autoimmune thyroid diseases," Arthritis Research \& Therapy, vol. 11, no. 4, article R106, 2009.

[36] N. Ishido, N. Inoue, M. Watanabe, Y. Hidaka, and Y. Iwatani, "The relationship between skewed X chromosome inactivation and the prognosis of Graves' and Hashimoto's diseases," Thyroid, vol. 25, no. 2, pp. 256-261, 2015.

[37] M. Kerkhof, D. S. Postma, B. Brunekreef et al., "Toll-like receptor 2 and 4 genes influence susceptibility to adverse effects of traffic-related air pollution on childhood asthma," Thorax, vol. 65, no. 8, pp. 690-697, 2010.

[38] G. Shi, T. Wang, S. Li et al., "TLR2 and TLR4 polymorphisms in southern Chinese psoriasis vulgaris patients," Journal of Dermatological Science, vol. 83, no. 2, pp. 145-147, 2016.

[39] W. K. Cho, M. H. Jung, S. H. Park et al., "Association of MICA alleles with autoimmune thyroid disease in Korean children," International Journal of Endocrinology, vol. 2012, Article ID 235680, 7 pages, 2012. 


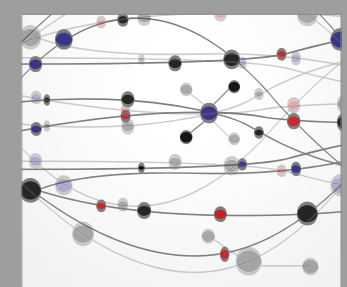

The Scientific World Journal
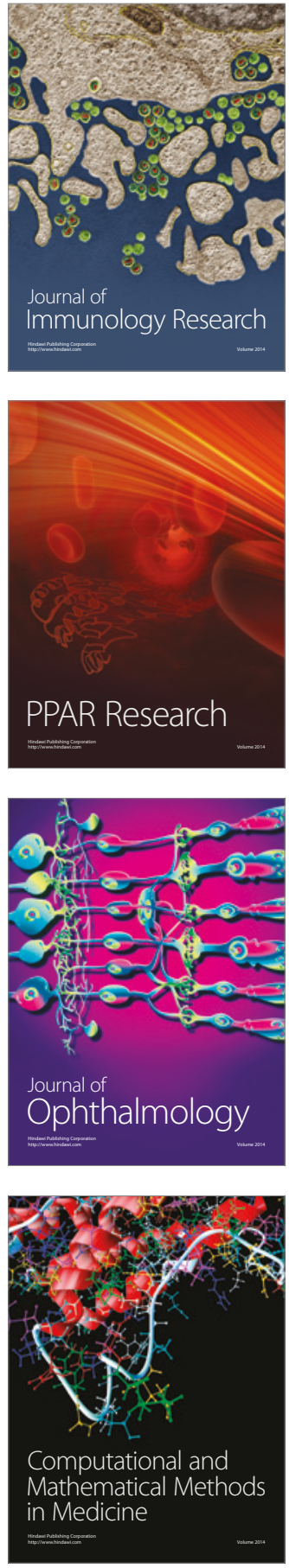

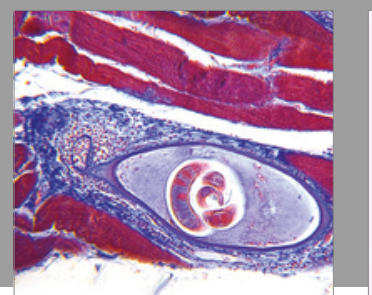

Gastroenterology Research and Practice
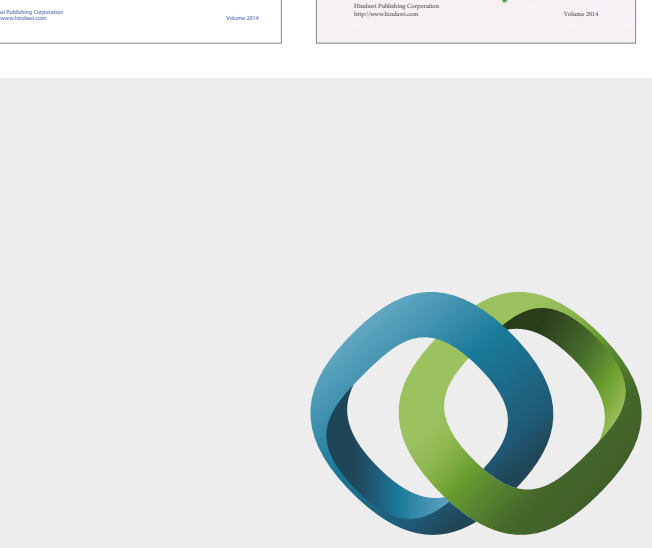

\section{Hindawi}

Submit your manuscripts at

https://www.hindawi.com
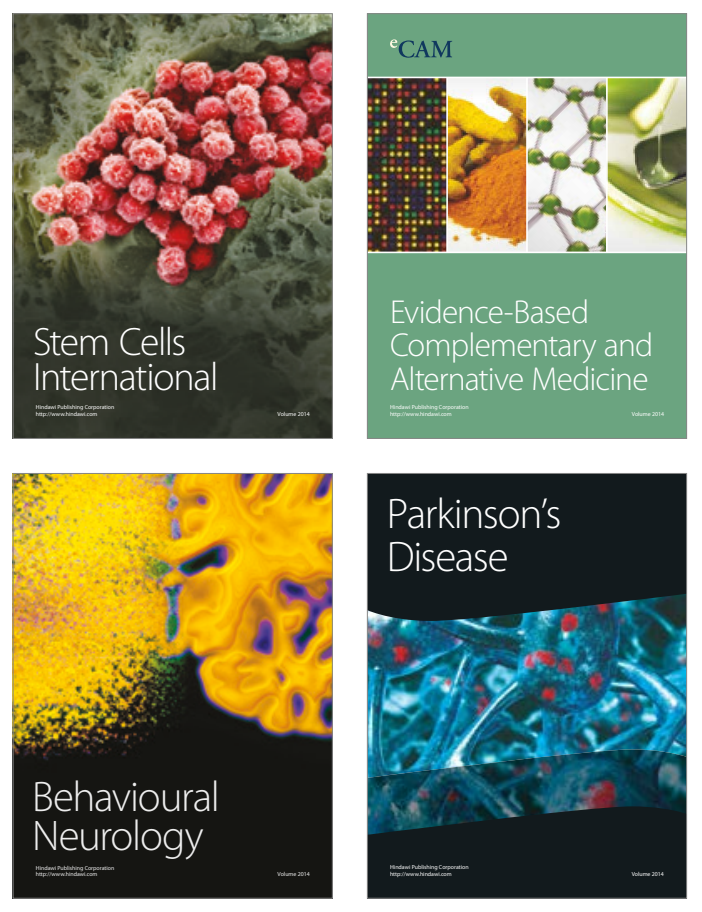
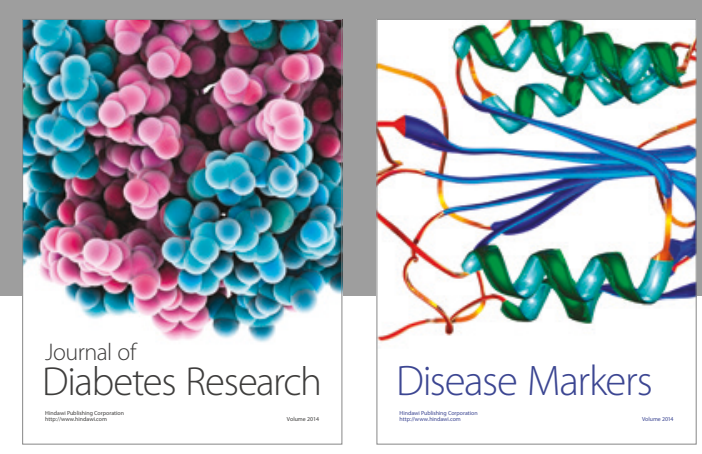

Disease Markers
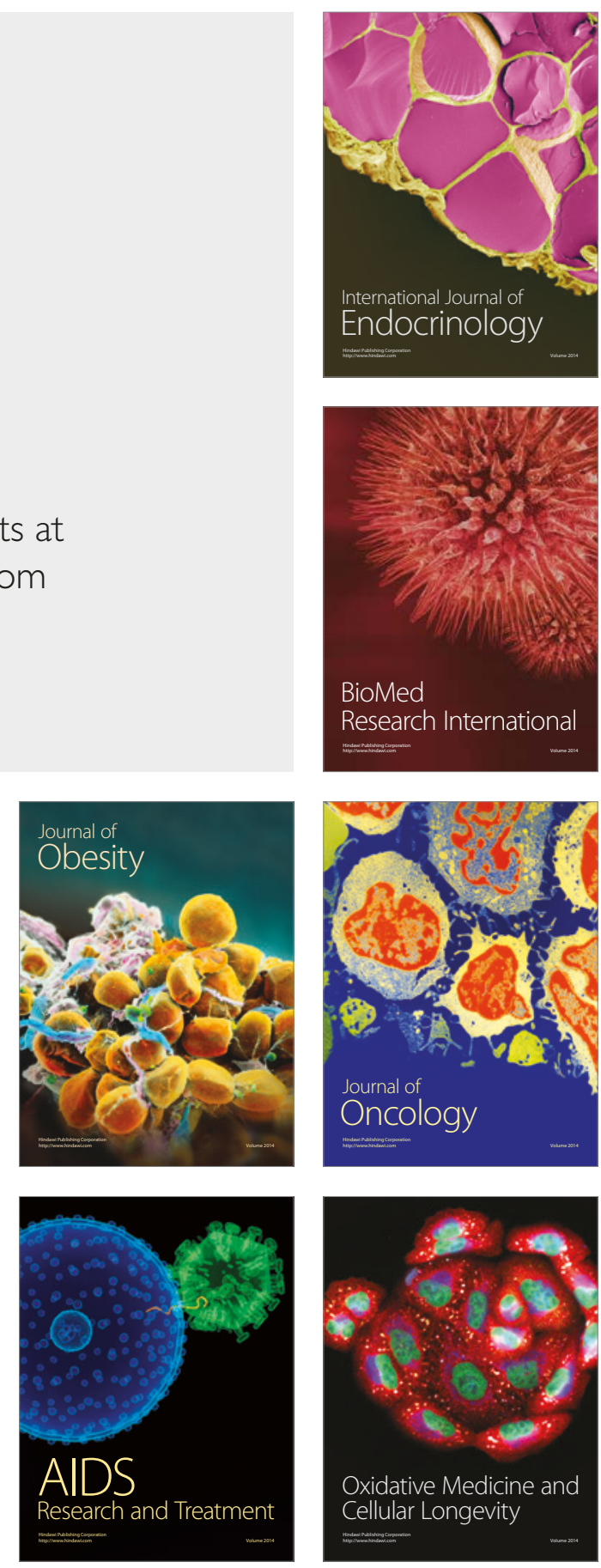